\title{
Virilization in a pregnancy
}

\author{
M W S Niranjala ${ }^{1}$, C N Wijeyaratne ${ }^{2}$, G K C Jayalath ${ }^{3}$ \\ Sri Lanka Journal of Diabetes, Endocrinology and Metabolism 2012; 2: 43-45
}

\begin{abstract}
We describe a 29-year old pregnant woman with history of primary hypothyroidism and impaired glucose tolerance presenting in her third trimester with preeclampsia and virilization. Initial investigations revealed markedly elevated serum testosterone, 17 hydroxy-progesterone and a non-suppressed serum cortisol following overnight dexamethasone. Imaging of the adrenals proved normal. At lower segment caesarean section, she had markedly enlarged ovaries with multiple haemorrhagic cysts; compatible with hyperreactio luteinalis. At two months postpartum, there was complete resolution of biochemical hyperandrogenemia with normalization of ovaries. Other causes of virilization such as late onset congenital adrenal hyperplasia, Cushing's syndrome and placental aromatase deficiency were excluded.
\end{abstract}

\section{Introduction}

Virilization during pregnancy is uncommon. This can manifest as clitoral enlargement, increased muscle strength, acne, hirsutism, frontal hair thinning and deepening of voice. Possible causes include the polycystic ovary syndrome (PCOS), androgen-producing tumours of the ovaries and adrenal glands, hypothyroidism, anabolic steroid exposure, late-onset congenital adrenal hyperplasia due to 21-hydroxylase deficiency and Cushing's syndrome. Aromatase deficiency, luteoma and hyperreactio luteinalis are rare conditions that can cause virilization during pregnancy (1).

\section{Case report}

A 29-year old nursing officer was transferred from a regional hospital at 31 weeks of gestation for investigation and management of high blood pressure and marked virilization. She noticed deepening of her voice and increased hair growth of her body since 20 weeks. On examination, she had excessive body hair with Ferriman Gallway score of 32/36, increased pigmentation and a deep husky voice. Her clitoris was markedly enlarged with a clitoral index of $49 \mathrm{~mm}^{2}$ (normal $<35 \mathrm{~mm}^{2}$ ). She had facial acne and acanthosis nigricans. There was no proximal muscle weakness, wasting, thin skin or easy bruising. She had striae gravidarum. She was clinically euthyroid with no features of acromegaly.

She had a first trimester spontaneous miscarriage in 2006. During next 3 years, she failed to conceive and was managed as for PCOS and had undergone 6 cycles of ovulation induction with clomiphene citrate although her current pregnancy was a spontaneous conception. She did not develop any features of virilization during clomiphene citrate treatment. She also had primary hypothyroidism and impaired fasting plasma glucose for which she was on treatment. Biochemistry revealed elevated plasma testosterone $16 \mathrm{ng} / \mathrm{mL}$ (normal 0.15 - 0.8 $\mathrm{ng} / \mathrm{ml}$ ), non suppressed overnight dexamethasone test (cortisol 6 micg / dL), suboptimal thyroid function when taking thyroxine 150 microgram daily (TSH $4.05 \mathrm{mIU} / \mathrm{mL}$ (0.4 - $4.5 \mathrm{mIU} / \mathrm{ml})$ Free T35.5pg/mL (1.4 - $4.4 \mathrm{pg} / \mathrm{mL})$ Free T4 0.76 ng/dL (0.8 - $1.8 \mathrm{ng} / \mathrm{dL})$, markedly elevated basal 17 OHP (58.5 ng/mL - normal 2-12 ng/mL) and impaired fasting plasma glucose $(5.9 \mathrm{mmol} / \mathrm{L})$. Ultrasound scan showed normal adrenal glands and a date compatible live fetus. Magnetic resonance imaging (MRI) of abdomen and pelvis was normal, but the adrenal glands were poorly visualized due to gravid uterus.

Possible aetiologies considered for hyperandrogenemia at this stage were late onset congenital adrenal hyperplasia, Cushing's syndrome, androgen secreting neoplasms of either ovaries or adrenals, and conditions specifically seen during pregnancy such as luteoma, hyperreactio luteinalis and placental aromatase deficiency.

At 34 weeks of pregnancy, she developed gross pedal edema, uncontrolled hypertension and proteinuria. Her liver enzymes and serum uric acid level started rising. Platelet count was reduced to $45,000 / \mathrm{mm}^{3}$. An emergency lower segment caesarean section was performed due to impending preeclampsia and HELLP syndrome. She delivered a live healthy baby girl weighing $1.840 \mathrm{~kg}$. During surgery, bilateral enlarged ovaries $(6 \times 4 \times 3 \mathrm{~cm})$ with multiple haemorrhagic cysts were noted (Figure 1).

${ }^{1}$ Consultant Endocrinologist, Teaching Hospital, Anuradhapura, ${ }^{2}$ Professor in Reproductive Medicine, Department of Obstetrics and Gynaecology, Faculty of Medicine, University of Colombo, ${ }^{3}$ Honorary Consultant Physician, De Soysa Hospital for Women, Colombo, Sri Lanka. 
Macroscopic appearance of the ovaries during caesarian section

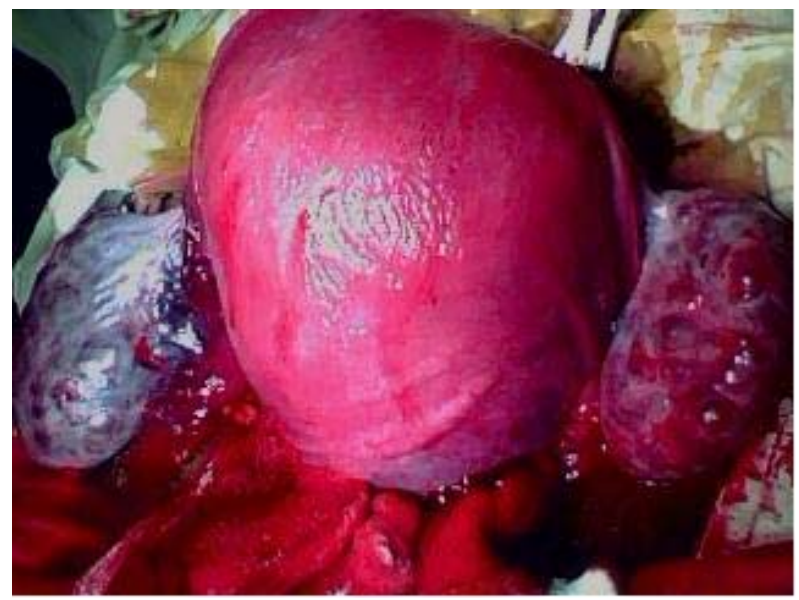

Figure 1. Bilateral enlarged ovaries $(6 \times 4 \times 3 \mathrm{~cm})$ with multiple hemorrhagic cysts.

Her immediate post partum period was uneventful. Histopathology of her placenta and umbilical cord was normal.

Her hormones were re-tested postpartum at 2 and 18 months. Her thyroid hormones were normal.

\begin{tabular}{llll}
\hline & $\begin{array}{l}\text { On } \\
\text { admission }\end{array}$ & $\begin{array}{l}2 \text { months } \\
\text { postpartum }\end{array}$ & $\begin{array}{c}18 \text { months } \\
\text { postpartum }\end{array}$ \\
\hline $\begin{array}{l}\text { Testosterone } \\
(\mathrm{ng} / \mathrm{mL})\end{array}$ & 16 & 0.2 & 0.22 \\
$\begin{array}{l}17 \mathrm{OHP} \\
(\mathrm{nmol} / \mathrm{L})\end{array}$ & 58.5 & $\begin{array}{l}\text { Basal }-1.0 \\
\text { Post ACTH }-1.5\end{array}$ \\
$\begin{array}{l}\text { ODST cortisol } \\
(\mathrm{micg} / \mathrm{dL})\end{array}$ & 1.7 & & \\
\hline
\end{tabular}

She had marked improvement of hirsutism. The voice became softer and the blood pressure remained normal. Ultrasound scan showed normal ovaries and adrenal glands at 4 months following delivery. At 18 months, she was totally free from all symptoms and signs of virilization except the mild persistent clitoromegally.

\section{Discussion}

This patient's history suggests a condition specific to pregnancy. The post partum normalization of hyperandrogenaemia supports our hypothesis that the virilization is caused by pregnancy. Following her delivery, the 60-minute corticotropin test for 17 - OHP excluded Non Classic Congenital Adrenal Hyperplasia (NC - CAH) in view of the normal basal value and post stimulation value < $45.5 \mathrm{nmol} / \mathrm{L}$ (2). During pregnancy, estrogen-dependent elevation of serum cortisol binding globulin (CBG), increases plasma cortisol and ACTH lead to a 2 - to 3-fold increment in plasma cortisol and the urinary free cortisol excretion (UFC). This complicates the screening process for Cushing's syndrome during pregnancy. Therefore, dexamethasone suppression test has limited utility during pregnancy with a high falsepositive rate (3). The normalized suppression test following delivery excludes Cushing's.

Having excluded late onset congenital adrenal hyperplasia and Cushing's syndrome, the possible causes for her presentation were aromatase deficiency, hyper reacteo luteinalis and luteoma.

Placental aromatase deficiency is rare. Active human placental aromatization of androgens protects the fetus against the virilizing action of fetal androgens. In the female fetus, this is particularly important to avoid the androgen effects on the differentiation of external genitalia. In aromatase deficiency, female babies will be born with ambiguous genitalia (4). Hence, the normal external genitalia of her baby daughter made aromatase deficiency most unlikely.

Hyperreactio luteinalis and luteoma of pregnancy are two different conditions that can cause maternal virilization in pregnancy. Elevated serum testosterone levels, $\delta 4$ androstenedione, and $5 \alpha$-dihydrotestosterone can be detected in patients with virilization $(5,6)$.

Luteoma of pregnancy are commonly unilateral but can be bilateral and multinodular. They are usually composed of large cells with abundant eosinophilic cytoplasm; they often have mitotic activity leading to erroneous diagnosis of malignant tumour. Luteoma represents an unusual response of the ovarian stromal cells to the altered hormonal levels of pregnancy (5). Conversely, hyperreactio luteinalis is typically associated with an abnormally high level of human chorionic gonadotropin, and is almost always bilateral and multicystic. In contrast to a luteoma of pregnancy, which is typically seen at or near term, hyperreactio luteinalis occurs at any time during pregnancy. Both lesions regress post partum $(5,6)$. Generally, these masses are discovered incidentally during caesarean delivery or tubal ligation. Some women develop hirsutism or virilization during late pregnancy with or without fetal masculinization due to circulating androgens. It is believed that the placental aromatization of androgens may function as a metabolic barrier that protects the fetus from effects of excessive maternal androgens, as in our patient's baby. Another protective mechanism may be the increased fetal exposure to potent estrogens, which may buffer the influence of androgens that reach the fetus (7).

Potential complications of hyperreactio luteinalis include ovarian torsion, infarction, and haemorrhage. The 
bilateral ovarian cysts usually regress shortly after pregnancy, and therefore, observation is typically the only required management unless complications occur $(5,6)$.

Other complications that have been observed are thyroid dysfunction, hyperemesis gravidarum, pre eclampsia (PE), intrauterine growth restriction, and delayed lactation. The elevation of hCG derived from placenta in a normal pregnancy could be caused by poor placentation, which could lead to the development of bilateral enlarged ovaries and a severe imbalance of angiogenic factors which can lead to development of preeclampsia (8).

In view of the above we conclude that her presentation is more in favour of hyperreactio luteinalis; which could have been confirmed by ovarian biopsy at delivery.

\section{References}

1. Manganiello, Paul D, Adams, Lisa V, Robert D, Ornvold K. Virilization during pregnancy with spontaneous resolution postpartum: a case report and review of the English literature. Obstetrical and Gynecological Survey 1995; 50(5): $404-10$.

2. Azziz R, Dewailly D, Owerbach D. Clinical review: nonclassic adrenal hyperplasia: current concepts. $J$ Clin Endocrinol Metab 1994; 78 (4): 810 - 15.

3. Lindsay JR, Nieman LK. The hypothalamic-pituitaryadrenal axis in pregnancy: challenges in disease detection and treatment. Endocrine Reviews 2005; 26 (6): 775 - 99.

4. Belgorosky A, Guercio G, Pepe C, Saraco N, Rivarola MA. Genetic and clinical spectrum of aromatase deficiency in infancy, childhood and adolescence. The Journal of Clinical Endocrinology and Metabolism 2009; 82(6): 321-30.

5. Ugaki H, Enomoto T, Tokugawa Y, Kimura T. Luteomainduced fetal virilization. Journal of Obstetrics and Gynaecology Research 2009; 35(5): 991-3.

6. Sarah MP, Jennie O'Brien. Hyperreactio luteinalis with clitoromegaly in a twin pregnancy. Obstet Gynecol 2009; 2(1): $3-4$.

7. Hensleigh PA, Carter RP, Grotjan HE Jr. Fetal protection against masculinization with hyperreactio luteinalis and virilization. The Journal of Clinical Endocrinology and Metabolism 1975; 40: 816- 23.

8. Masuyama H, Tateishi Y, Matsuda M, Hiramatsu Y. Hyperreactio luteinalis with both markedly elevated human chorionic gonadotropin levels and an imbalance of angiogenic factors subsequently developed severe earlyonset preeclampsia. Fertil Steril 2009; 92(1): 393 -4. 